
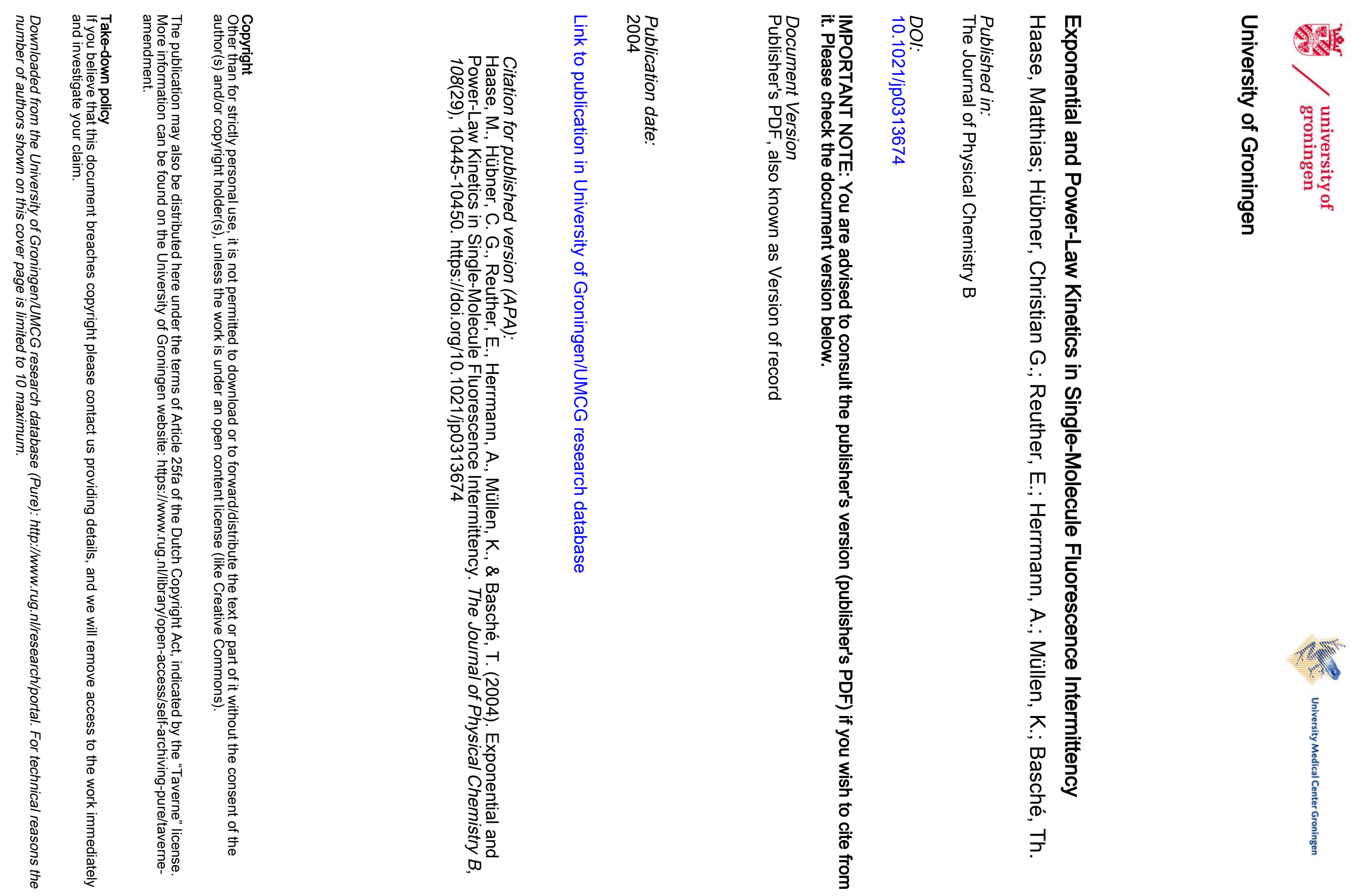


\title{
Exponential and Power-Law Kinetics in Single-Molecule Fluorescence Intermittency ${ }^{\dagger}$
}

\author{
Matthias Haase, ${ }^{\ddagger}$ Christian G. Hiibner, ${ }^{*}$ E. Reuther, ${ }^{\S}$ A. Herrmann,, K. Miillen, ${ }^{\S}$ and \\ Th. Basché ${ }^{\dagger}$ \\ Institut für Physikalische Chemie, Universität Mainz, Jakob-Welder-Weg 11, 55128 Mainz, Germany, and \\ Max-Planck-Institut für Polymerforschung, 55128 Mainz, Germany.
}

Received: December 31, 2003; In Final Form: March 24, 2004

\begin{abstract}
Perylenemonoimide chromophores, attached to a small dendron arm embedded in the polymer hosts Zeonex, poly(vinylbutyral), and poly(methyl methacrylate), are studied by means of laser-induced confocal fluorescence detection. Transient fluorescence intensity exhibits dark periods on two different time scales: on a $100 \mu \mathrm{s}$ time scale and on a considerably longer time scale, ranging from $100 \mathrm{~ms}$ to as much as tens of seconds under high-vacuum conditions. The exponentially distributed short "off" times are attributed to triplet excursions of the molecule. The long-lived dark states follow a power-law distribution and are discussed in terms of the formation of radical anions/cations via electron tunneling processes.
\end{abstract}

\section{Introduction}

One of the most intriguing and most intensely studied features of single molecule fluorescence is fluorescence intermittency, i.e., the reversible conversion into a dark state, often referred to as "on"-"off" blinking. ${ }^{1}$ This phenomenon can be observed on time scales from a few microseconds to tens of seconds. Triplet shelving, first observed at cryogenic temperatures, ${ }^{2,3}$ was identified as one mechanism leading to reversible dark states in the fluorescence of single dye molecules and therefore to the emission of photons in bunches. In the following years, photon bunching at ambient conditions has been the subject of numerous studies, ${ }^{4-7}$ where the influence of the environment, particularly the oxygen quenching effect, was most intensely investigated, and triplet lifetimes ranging from microseconds up to $100 \mathrm{~ms}$ were reported..$^{5,8-11}$

In contrast to the well-understood triplet mechanism, the nature of slow blinking of single molecules on a millisecond to second time scale is not unambiguously recognized yet. Depending on the system under investigation, several models are discussed as being responsible for the occurrence of these long-lived dark states: (i) modification of the chromophore's conformation shifting the absorption away from the excitation or reducing the fluorescence quantum yield ${ }^{4,12,13}$ (ii) reversible photochemical processes like formation of an ionized ${ }^{7,14-17}$ or a metastable oxidized state, ${ }^{18,19}$ (iii) quenching by nearby molecules. ${ }^{7,14}$ The time scales of fluorescence intermittency attributed to the various processes are similar, requiring additional information in order to identify the underlying mechanism.

On the other hand, distinguishing triplet excursions from blinking due to other processes is complicated by the possible overlap of the time scales of fluorescence intermittency due to these other processes. In multichromophoric entities embedded in two different polymers, dark states of very similar duration

\footnotetext{
† Part of the special issue "Gerald Small Festschrift".

* To whom correspondence should be addressed: Universität Halle, Fachbereich Physik, Hoher Weg 8, 06120 Halle. E-mail: ch.huebner@ physik.uni-halle.de.

$¥$ Universität Mainz.

$\S$ Max-Planck-Institut für Polymerforschung.
}
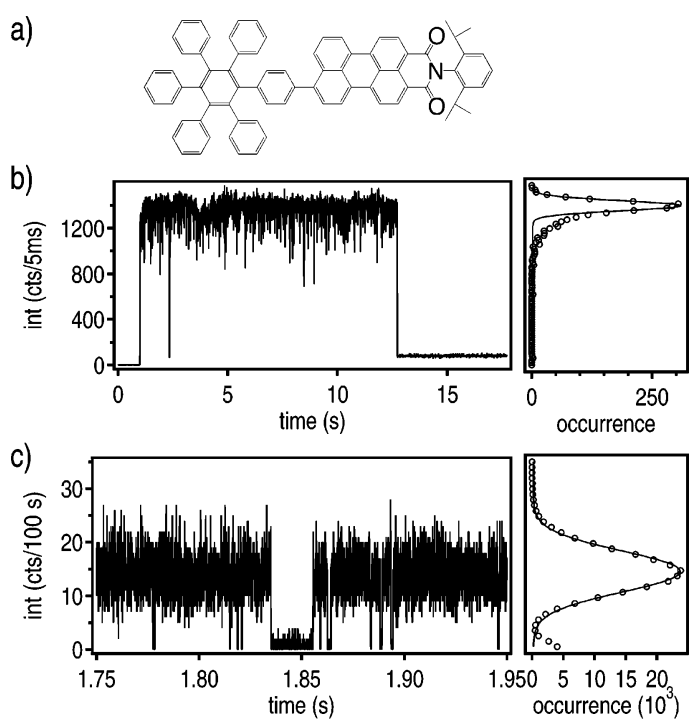

Figure 1. (a) Structure of the compound g0. (b and c) Transient fluorescence intensity of a single g0 molecule in Zeonex under vacuum. Part $\mathrm{b}$ shows the transient with a bin time of $5 \mathrm{~ms}$ and part $\mathrm{c}$ a blowup at about $1.85 \mathrm{~s}$ with a bin time of $100 \mu \mathrm{s}$. Periods of background fluorescence are visible in both traces. The right-hand panels in $\mathrm{b}$ and c show intensity histograms (circles) together with fitted Gaussians (lines). The molecule was excited with linearly polarized light at 488 $\mathrm{nm}$ with an intensity of $2 \mathrm{~kW} \mathrm{~cm}^{-2}$.

and frequency of occurrence were attributed in one case to triplet excursions ${ }^{20,21}$ and in the other case to the formation of an ionized state. ${ }^{22}$

The concern of the present study is a thorough investigation of the "on"-"off" behavior of a perylenemonoimide (PMI) model system on time scales ranging from several tens of microseconds to seconds in order to clearly identify the underlying photophysical processes. The model system named g0 in the following is a PMI attached to a small dendritic arm (see Figure 1a). In the experiment, we find in all three investigated polymer hosts short "off" times of g0 with an average duration between some $10 \mu \mathrm{s}$, if the sample is exposed to air, and few hundred microseconds under argon flow and in a vacuum. Additionally, longer-lived dark states with a duration 
ranging from milliseconds to seconds are observed. The short "off" times with a length on the $10-100 \mu$ s time scale, similar to that recently reported for a bichromophoric molecule where two PMI chromophores were linked by a benzil unit, ${ }^{23}$ can be attributed to triplet excursions. The duration of the long-lived dark states is not exponentially distributed but instead follows a power law. They may be explained by the formation of radical anions/cations due to charge tunneling, where the charge trapping rates are widely distributed.

On the basis of Monte Carlo simulations, we show that both methods for the determination of triplet lifetimes described in the literature, namely the fluorescence autocorrelation and the histogram method, are suited to reveal triplet lifetimes as short as half the binning time for the intensity transient under realistic experimental conditions.

\section{Experimental Section}

Thin-film samples of the model compound g0 (see Figure 1) were prepared by spin casting a drop of a toluene solution of the dye $(1 \mathrm{nM})$ and the polymer $(5 \mathrm{~g} / \mathrm{L})$ onto glass microscope coverslips at a rotational speed of $4000 \mathrm{~min}^{-1}$. Prior to spin casting the coverslips were cleaned by heating to $515{ }^{\circ} \mathrm{C}$ for 1 $\mathrm{h}$ in a furnace. The resulting films have a thickness between 10 and $30 \mathrm{~nm}$ and contain $\mathbf{g 0}$ at an areal density of $0.5 \mu \mathrm{m}^{-2}$. In a control experiment with a sample prepared exactly the same way but without g0, less than one fluorescent spot per $25 \mu \mathrm{m}^{2}$ was observed on average. Fluorescence excitation and detection was accomplished in a custom-built scanning confocal optical microscope (SCOM). A single-mode optical fiber spatially filtered the exciting laser light provided by a continuous-wave $\mathrm{Ar} / \mathrm{Kr}$ mixed gas laser (Melles Griot, Carlsbad, CA) operated at $488 \mathrm{~nm}$. An all-fiber polarization controller (General Photonics, Chino, CA) allowed for the adjustment of arbitrary states of polarization of the excitation light. The collimated beam is directed by a dichroic mirror to the back aperture of a N.A. 1.4 oil immersion microscope objective lens (Apochromat, Zeiss, Jena, Germany), which produces a nearly diffraction-limited spot (fwhm: $210 \mathrm{~nm}$ ) in the sample plane. Fluorescence collected by the microscope objective passes the dichroic mirror and is further filtered by a dielectric long pass filter (XR3000, Omega Filters, Brattleboro, VT) in order to suppress back-reflected laser light. A lens with a focal length of $40 \mathrm{~cm}$ focused the fluorescence onto a single photon avalanche photodiode (SPAD, SPCM AQ 14, Perkin-Elmer, Fremont, CA) for detection. The rather large focal length is necessary since the active area of the SPAD with a diameter of $180 \mu \mathrm{m}$ serves as the detection pinhole. The pulses from the APD were processed in a programmable digital signal processing (DSP) board (ADwin Light-16, Jäger, Germany) that also supplies the control voltages for the $x-y$ piezo scanning stage (P-731.8C, Physik-Instrumente, Germany). Experiment control was realized by a computer program written in LabView (National Instruments, Austin, TX). In imaging mode a raster scanning image with a custom integration time per pixel can be taken. After positioning a single molecule of $\mathbf{g 0}$ in the confocal volume, transient intensities are recorded by counting the pulses from the APD into bins of custom width. To flush the sample with argon, a $2 \mathrm{~cm}$ wide tube was placed close to the sample surface. A custom-made sample holder allowed for reducing the pressure above the sample to $10^{-6}$ Torr. Data Analysis and simulations were performed with Igor Pro (Wavemetrics, Lake Oswego, OR).

\section{Results and Discussion}

Pers $\mathrm{b}$ and $\mathrm{c}$ of Figure 1 show transient intensities of a single g0 molecule embedded in a Zeonex film under conditions of

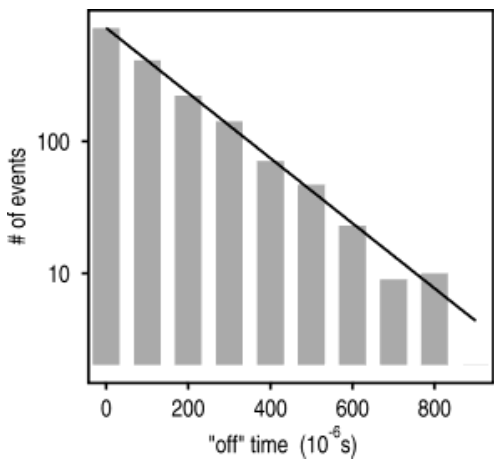

Figure 2. Histogram of "off" times built from the transient intensity shown in Figure 1. The line is an exponential fit to the histogram in the range from 0.1 to $1.5 \mathrm{~ms}$ enabling the determination of the average "off" time, which is $175 \mu$ s for this particular molecule.

high vacuum $\left(10^{-6}\right.$ Torr). Panels $\mathrm{b}$ and $\mathrm{c}$ both show the same transient intensity, but with different time resolution. Panel b represents the complete time trace with an integration time of $5 \mathrm{~ms}$, whereas panel $\mathrm{c}$ is a blow-up of a very short period of the whole transient with $100 \mu$ s integration time. Both traces show features characteristic for single-molecule fluorescence: discrete jumps between intensity levels and, in part b, one sudden photobleaching step to the background intensity level, i.e., the intensity if no molecule is in the focus.

In Figure 1c, two intensity levels are clearly distinguishable from the shot noise of photon counting, where the lower level equals the background intensity. Many periods of background intensity -in the following denoted as "off" times - with a short duration and one considerably longer are visible in part c. Contrarily, in Figure 1b, the "off" states appear to be rare events. However, intensity fluctuations toward lower levels exceeding shot noise are visible. The fluorescence intensity histogram shown right next to the timetrace in Figure $1 \mathrm{~b}$ reveals this deviation, as it is clearly asymmetric compared to the fitted Gaussian, which is a good approximation for the Poisson distribution of shot noise for higher mean values. Such an asymmetry can be regarded as a clear sign for unresolved blinking kinetics due to insufficient time resolution. The intensity histogram for the short bins in comparison shows no asymmetry and can be nicely fitted with a Gaussian for the "on" level. Inspecting Figure $1 \mathrm{~b}$, there is one event at about $1.8 \mathrm{~s}$ where the intensity drops to the background level. Here the question arises as to whether this event is caused by the same mechanism as the short "off" times. The difference in the time scales of the "off" times visible in panels $b$ and c becomes evident if one looks at the "off" event of panel b in the enlarged time scale of panel c. The "off" event at about $1.8 \mathrm{~s}$, which is visible as a sharp spike in panel $b$, is very long compared to the other "off" events visible in panel c. However, this visual difference needs to be rationalized. For this purpose, a histogram of the "off" times from the whole transient binned at $100 \mu \mathrm{s}$ was compiled, which is shown in Figure 2. In a semilogarithmic plot, the histogram is fairly well approximated by a straight line indicating an exponential distribution. The corresponding fit yields an average "off" time of $175 \mu$ s for this particular molecule. (i) We attribute the short "off" times to triplet excursions since the "off" times are exponentially distributed. (ii) This means, the process is dominated by a single rate, the quantum yield of the process leading to the "off" times is $5 \times$ $10^{-5}$ and therefore comparable to intersystem crossing (ISC) quantum yields found for a similar dye. ${ }^{5,22}$ The quantum yield was determined from histograms of the detected photons during "on" periods, ${ }^{9}$ (iii) The "off" times are shorter in the presence 

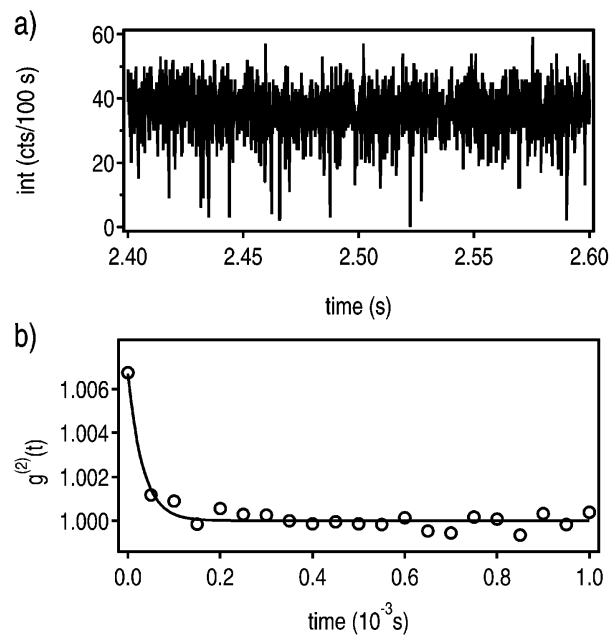

Figure 3. (a) Transient intensity of a single g0 molecule embedded in Zeonex exposed to air with a bin time of $100 \mu$ s. Spikes exceeding shot noise but not reaching the background level are visible. (b) Secondorder correlation function calculated from the transient intensity.

of oxygen as will be further elaborated in the following, which is expected because of the well-known oxygen quenching effect. ${ }^{9}$

Assuming that the $25 \mathrm{~ms}$ long off period visible in panels $b$ and $\mathrm{c}$ of Figure 1 belongs to the triplet process, the probability of its occurrence can be easily estimated by extrapolating the fit in Figure 2 to $25 \mathrm{~ms}$. The number obtained is on the order of $10^{-59}$, which makes it extremely unlikely that this "off" time belongs to the exponential distribution of the triplet "off" times, taking into account the total number of "off" times being $<10^{4}$. The long-lived dark states will be discussed later in this section. First, we want to focus on the triplet kinetics.

To study the influence of the environment, the transient intensities were recorded in three different polymers as well as in three environments, i.e., ambient air, argon, and high vacuum. Figure $3 \mathrm{a}$ shows the transient intensity of an individual g0 molecule embedded in Zeonex in ambient air. Comparing this transient to the one in Figure 1c the difference is clearly visible. There are many spikes of the transient that do not reach the background level indicating that there are unresolved "off" times. To estimate the triplet lifetime, we plot the second-order correlation function $g^{(2)}(t)$ in Figure $3 \mathrm{~b}$. In the case of two intensity levels with single rates for the transitions between both levels, $g^{(2)}(t)$ takes the form

$$
g^{(2)}(t)=1+C e^{-\left(k_{o n}+k_{o f f}\right) t}
$$

where $C$ is called molecular contrast and $k_{\text {on }}$ and $k_{\text {off }}$ are the rates to and from the "on" level. The molecular contrast and the respective rates are connected with the intensity during the "on" and "off" periods $I_{\text {on }}$ and $I_{\text {off }}$ via ${ }^{24}$

$$
C=\frac{k_{\mathrm{on}} k_{\mathrm{off}}\left(I_{\mathrm{on}}-I_{\mathrm{off}}\right)^{2}}{\left(k_{\mathrm{on}} I_{\mathrm{on}}+k_{\mathrm{off}} I_{\mathrm{off}}\right)^{2}}
$$

which can be further simplified if $I_{\text {on }} \gg I_{\text {off: }}$ :

$$
C=\frac{k_{\mathrm{off}}}{k_{\mathrm{on}}}
$$

These expressions generally require that the integration time is short compared to the "on" and "off" times, respectively. This requirement is not fulfilled for the "off" times in Figure 3a
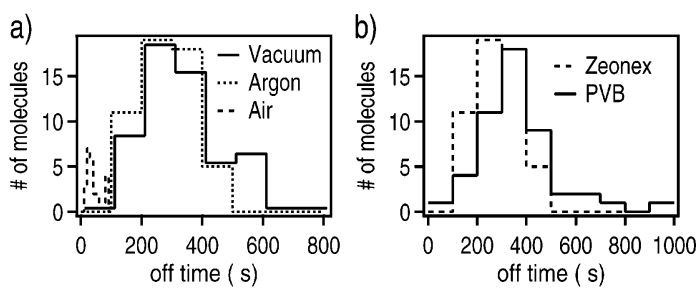

Figure 4. Histograms over the average "off" times of 50 investigated g0 molecules under different conditions. In part a the molecules are embedded in Zeonex and the atmosphere was varied, whereas in part $\mathrm{b}$ the molecules were measured under argon atmosphere in two different polymers.

because the "off" times obviously do not exceed the integration time. However, the "off" time can still be determined with sufficient accuracy, as the analysis of the Monte Carlo simulations in the following section will show. From $g^{(2)}(t)$ in Figure $3 \mathrm{~b}$ an "off" time of $32 \mu \mathrm{s}$ is derived by fitting an exponential decay and using eqs 1 and 3. This value is considerably shorter compared to the triplet lifetime of $\mathbf{g 0}$ under vacuum.

To study the influence of oxygen, 50 molecules of $\mathbf{g} \mathbf{0}$ in each case embedded in Zeonex were investigated in a vacuum, argon, and ambient air. The results are shown as histograms in Figure 4a. A considerable number of molecules investigated in air underwent rapid photodestruction preventing the determination of the triplet lifetime; therefore, the corresponding histogram contains only a total of 22 molecules. It peaked at $30 \mu \mathrm{s}$, and the average "off" time in air is $48 \mu \mathrm{s}$. The width of this distribution mainly reflects the experimental uncertainty. Additionally, one has to notice that the average value may be biased by the limitations in time resolution and observation time. For extremely short "off" times compared to the "on" times, the contrast $C$ in eq 1 turns out to be very small, which renders a fit to the experimental data impossible.

In a vacuum as well as in argon all molecules were stable long enough to determine the triplet lifetime precisely. The offtime histograms for argon and vacuum match and are peaked around $300 \mu \mathrm{s}$. Here, the width of the histograms is no longer determined by the experimental error. Thus, we conclude that there is a distribution of triplet lifetimes, which can be attributed to heterogeneity in the sample. A possible explanation assumes structural flexibility, leading to a change in the spin-orbit coupling, ${ }^{9}$ and it will be elaborated on in more detail in a forthcoming work.

Comparing the histograms in Figure $4 \mathrm{a}$, the quenching effect due to oxygen is evident. The noticeable similarity of the histograms in argon and vacuum allows the conclusion that the oxygen concentration relevant for triplet quenching is comparably low in both cases, under argon flow and in a vacuum.

The structural flexibility may also depend on the host material. Therefore, experiments in argon atmosphere were performed for a different host polymer, poly(vinylbutyral) (PVB). In Figure $4 \mathrm{~b}$ the "off" time histograms of 50 molecules investigated under argon in Zeonex and PVB, respectively, are shown. The histogram for PVB is shifted to longer times making evident the influence of the matrix on the triplet lifetime. Since both polymers, Zeonex and PVB, do not contain heavy atoms, the heavy-atom effect cannot be responsible for the differing triplet lifetime. Additionally, the variations within one polymer are larger than the shift between both polymers, i.e., structural variations appear to be predominantly responsible for the triplet lifetime variations. The average triplet lifetimes of $\mathbf{g} \mathbf{0}$ in different matrices and atmospheres are summarized in Table 1. The oxygen quenching effect is evident for the three host materials. 
TABLE 1: Average Triplet Lifetimes in $\mu$ s for G0 in Three Different Host Materials and Atmospheres

\begin{tabular}{lccc}
\hline & Zeonex & PVB & PMMA \\
\hline air & 50 & 110 & 170 \\
argon & 280 & 360 & 450 \\
vacuum & 310 & $a$ & $a$
\end{tabular}
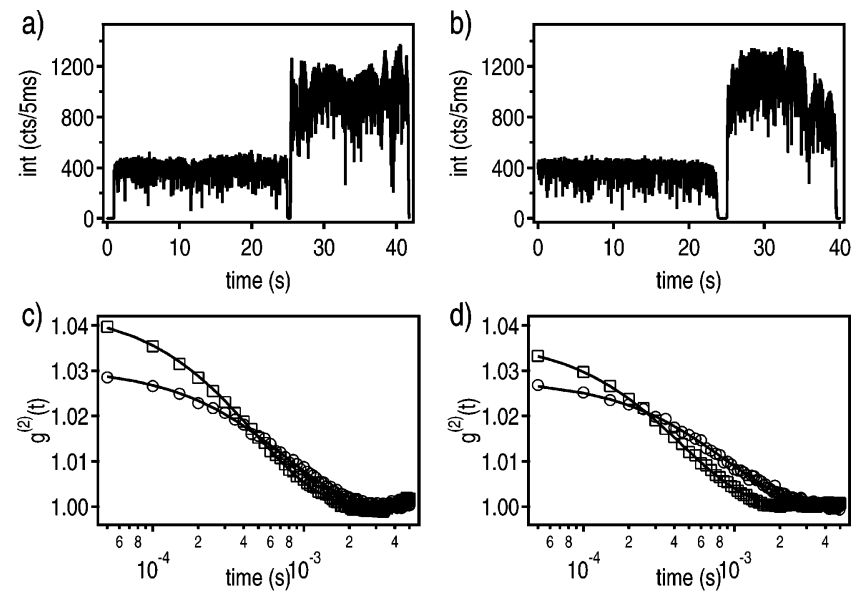

Figure 5. ( $a$ and $b$ ) Transient intensities of two g0 molecules embedded in Zeonex under argon flow. The first $\approx 22 \mathrm{~s}$ were recorded at a laser intensity of $0.5 \mathrm{kWcm}^{-2}$, subsequently the laser intensity was increased to $1.5 \mathrm{kWcm}^{-2}$. (c and d) Autocorrelation functions corresponding to the lower intensity (circles) and higher intensity (squares). The lines are exponential fits yielding "off" times of $0.75 / 0.51 \mathrm{~ms}$ for one molecule and $0.89 / 0.46 \mathrm{~ms}$ for the other molecule at the lower/higher intensity.

For two molecules embedded in Zeonex fluorescence transients were recorded at two different laser intensities, i.e., 0.5 and $1.5 \mathrm{~kW} \mathrm{~cm}^{-2}$. The transient intensities and the autocorrelation functions in the respective time windows are shown in Figure 5. In both cases, a shortening of the triplet lifetime at higher excitation rates is observed, from $\approx 1 \mathrm{~ms}$ at the lower laser intensity to $\approx 0.5 \mathrm{~ms}$ at the higher laser intensity. Power shortening of the triplet lifetime was reported for terrylene embedded in $p$-terphenyl and attributed to a reverse ISC process via higher excited triplet states. ${ }^{25}$ Therefore, we conclude that the observed shortening is due to pumping into higher excited triplet states as discussed by Fleury et al. ${ }^{25}$ This power dependence is possibly responsible for the generally shorter triplet lifetimes found here as compared to the recently reported values of a very similar compound. ${ }^{22}$

In the remainder of this section, we want to address the longlived dark states. Unfortunately, these dark states are rare events, thus in conjunction with the limited photostability of the g0 molecules rendering it impossible to register a sufficient number of long-lived dark states from a single molecule. No distribution can thus be generated on a single-molecule basis. It turned out that long-lived dark states are more frequently observed in another host polymer, poly(methyl methacrylate) (PMMA). Thus, by combining the transient intensities of 40 molecules measured in a vacuum in PMMA, a distribution of "off" times can be compiled as shown in Figure 6a. At first we notice the remarkable time range of "off" times over more than 1 decade, which is incompatible with a single exponential distribution. In a semilogarithmic plot, the distribution does not appear as a straight line but rather as a bent curve, again indicating deviations from single-exponential behavior. If we plot the histogram doubly logarithmic, we find a straight line, which is indicative of a power law distribution. From a fit to the
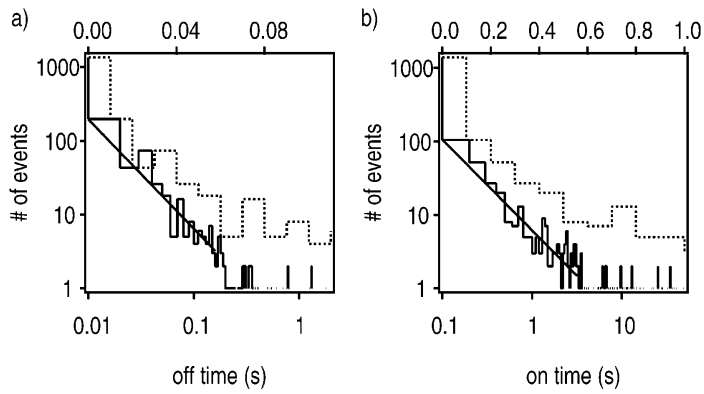

Figure 6. (a) "Off" time histogram as compiled from 40 single g0 molecules. The solid and dotted curves represent $\log -\log$ (bottom axis) and semilog plots (top axis) of the same histogram, respectively. The line is a power-law fit to the histogram, giving rise to an exponent of -1.5. (b) Corresponding histogram of the "on" times. Here, the exponent in the power law is -1.25 .

distribution we find an exponent of -1.5 . For semiconductor nanocrystals (NCs), off-time distributions following a power law with exponents between -1.37 and -1.79 were reported. ${ }^{26,27}$ Charge tunneling processes are proposed as the origin of the dark states in semiconductor NCs. The main difference in "on"-_off" behavior between the semiconductor NCs and the molecules in the present study is the considerably lower frequency of occurrence of the "off" states in our case. The duration of "on" times is also stretched over more than one decade as shown in Figure 6b, and by fitting with a power-law we find an exponent of -1.25 . In general, a power-law behavior of the "on" and "off" time duration is qualitatively inconsistent with a single transition rate to and from a dark state, but consistent with a distribution of transition rates over a wide range. As pointed out by Kuno et al., ${ }^{26}$ a distribution of rates spanning orders of magnitude can be straightforwardly explained by tunneling processes with a fluctuating barrier width. This is in accordance with the proposed mechanism of radical ion formation $^{7}$ for the blinking of single molecules since the ions may be formed and recovered by a charge tunneling process. Recently the formation of a radical anion could be directly demonstrated in ensemble experiments for a Rhodamine dye ${ }^{28}$ and for a dendrimer bearing two PMI chromophores at the rim. ${ }^{22}$ However, there is no direct evidence for radical ion formation from our experiments. The observation that the long "off"-times are more frequent in PMMA as compared to Zeonex could be a hint into this direction. A radical ion may be formed and stabilized more easily in the more polar PMMA host.

An additional peculiarity results from building the histogram from many molecules: We cannot decide whether the distribution of rates is static, i.e., where the rates differ from molecule to molecule, or dynamic, i.e., where the rate fluctuates in time. We would like to stress that if those fluctuations happen on the same time scale as the rate itself, the description by a rate fluctuating in time is not appropriate. Much longer time traces were needed to answer this question, which would require even more photostable chromophores. For a similar chromophore an exponential distribution of "off" times attributed to an ionic state was recently reported. ${ }^{29}$ In this study, however, the molecules where placed on self-assembled monolayers on top of an indium-tin oxide electrode with a well-defined distance between the chromophore and the charge-trapping center.

It has to be emphasized that the tunneling process causes a few short "off" times with a comparable duration as the triplet "off" times because of the inherently wide time span of a power law. On the other hand, triplet "off" times lasting for $100 \mathrm{~ms}$ are extremely unlikely to be observed within the time window of our experiments. 
TABLE 2: Results of the Analysis of Monte Carlo Simulated Intensity Transients as Obtained from Two Different Methods, the Autocorrelation Method and the Histogram Method ${ }^{a}$

\begin{tabular}{|c|c|c|c|c|c|c|c|c|c|}
\hline & & \multicolumn{4}{|c|}{ autocorrelation } & \multicolumn{4}{|c|}{ histograms } \\
\hline \multicolumn{2}{|c|}{ input } & \multicolumn{2}{|c|}{ off $t(\mu \mathrm{s})$} & \multicolumn{2}{|c|}{ on $t(\mathrm{~ms})$} & \multicolumn{2}{|c|}{ off $t(\mu \mathrm{s})$} & \multicolumn{2}{|c|}{ on $t(\mathrm{~ms})$} \\
\hline $\begin{array}{l}\text { off } t \\
(\mu \mathrm{s})\end{array}$ & $\begin{array}{l}\text { on } t \\
\text { (ms) }\end{array}$ & $\begin{array}{c}50 \mu \mathrm{s} \\
\mathrm{bin}\end{array}$ & $\begin{array}{c}100 \mu \mathrm{s} \\
\text { bin }\end{array}$ & $\begin{array}{c}50 \mu \mathrm{s} \\
\mathrm{bin}\end{array}$ & $\begin{array}{c}100 \mu \mathrm{s} \\
\text { bin }\end{array}$ & $\begin{array}{c}50 \mu \mathrm{s} \\
\mathrm{bin}\end{array}$ & $\begin{array}{c}100 \mu \mathrm{s} \\
\text { bin }\end{array}$ & $\begin{array}{c}50 \mu \mathrm{s} \\
\text { bin }\end{array}$ & $\begin{array}{c}100 \mu \mathrm{s} \\
\text { bin }\end{array}$ \\
\hline 50 & 1 & $48 \pm 2$ & $48 \pm 5$ & $2.9 \pm 0.1$ & $6.3 \pm 0.6$ & $48 \pm 1$ & $52 \pm 2$ & $2.8 \pm 0.1$ & $8.5 \pm 0.4$ \\
\hline 300 & 5 & $294 \pm 15$ & $290 \pm 13$ & $7.2 \pm 0.5$ & $8.5 \pm 0.5$ & $230 \pm 14$ & $290 \pm 23$ & $3.4 \pm 0.3$ & $7.3 \pm 0.3$ \\
\hline
\end{tabular}

${ }^{a}$ Two sets of input values were used reflecting the experimental values in air and without oxygen.

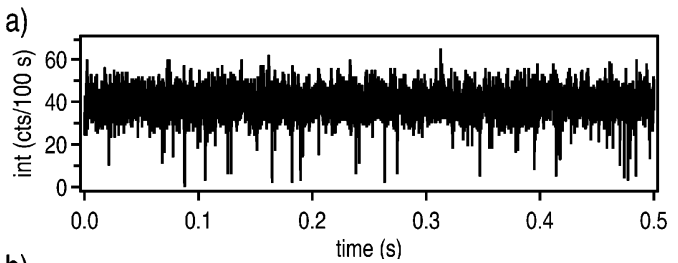

b)
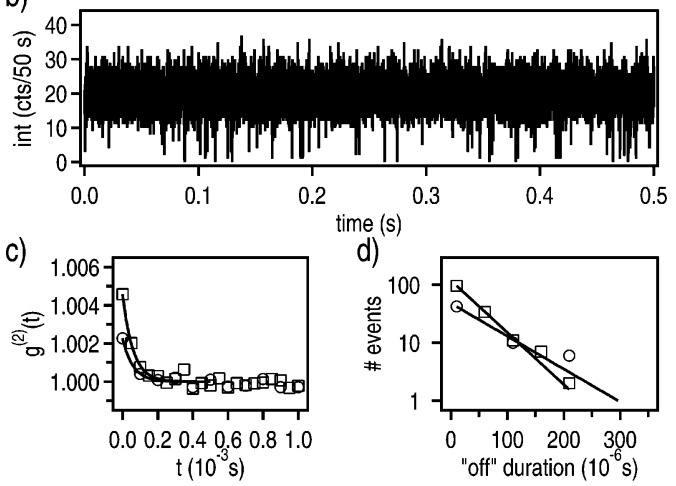

d)

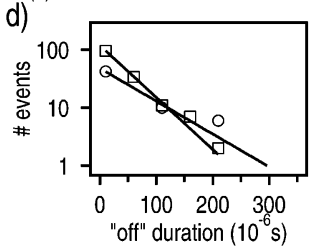

Figure 7. Simulated transient intensities obtained from a Monte Carlo method ( $a$ and $b$ ) and respective analysis ( $c$ and $d)$. The two traces represent identical data but sampled with different integration times. In panel a, the number of resolved "off" times is lower. Panels c and $\mathrm{d}$ visualize the analysis of the simulated data with the autocorrelation and histogram methods, respectively. Here, the circles represent the analysis of the $100 \mu$ s binned trace and the squares the analysis of the $50 \mu$ s binned trace.

Simulations. A requirement for proper determination of "on"-"off" kinetics from the autocorrelation function (ACF) is a short bin time compared to the waiting time in the "on" and "off" states, respectively. ${ }^{12}$ On the other hand, the histogram method is in principle able to accurately reveal the average "off" time under the condition of longer bin times if the molecule can be observed sufficiently long. ${ }^{9}$ Because the bin times in the present investigation are comparable to the "off" time duration, we test by the aid of Monte Carlo simulations whether the determination of the "off" time with the ACF and histogram method is still appropriate under these conditions. Transient intensities of $5 \mathrm{~s}$ duration were simulated where the outcome of the analysis of the experimental data was used as input parameters. "On" and "off" times were generated by the transformation of uniformly distributed random numbers into exponentially distributed ones. Subsequently, the number of counts per time bin in the "on" and "off" parts of intensity traces were drawn from Poisson distributions with mean values corresponding to the average count rates found in the experiment. Figure 7 shows examples of simulated intensity transients. Panels a and b both represent identical transients, but sampled at different integration times of 100 and $50 \mu \mathrm{s}$, respectively. The parameters are $50 \mu \mathrm{s}$ "off" time, $1 \mathrm{~ms}$ "on" time, and count rates of $400 \mathrm{kHz}$ for the "on" state and $10 \mathrm{kHz}$ for the background, respectively. The intensity traces were first simulated for a bin time of $10 \mu \mathrm{s}$ and subsequently resampled with the above-mentioned integration times. For the analysis of the "on"-"off" kinetics, the two methods mentioned above were chosen, i.e. calculation of the second-order correlation function $g^{(2)}(t)$ and compilation of "on"- and "off"-time histograms. While for the determination of "on"-"off" kinetics by the ACF and its the interpretation via eq 1 the assumption of actual "on""off" behavior is sufficient, the histogram method requires a threshold distinguishing between the "on" and the "off" level as an additional input parameter. This threshold was chosen to match the count rate where the Poisson distributions of the background and the "on" level cross. In principle, the threshold has to be adjusted with respect to the relation between "on" and "off" times, ${ }^{9}$ but we did not want to put prior knowledge into the analysis here.

From the autocorrelation analysis of one particular transient presented in Figure 7c, an "off" time of $\approx 50 \mu \mathrm{s}$ is obtained for both integration times, 50 and $100 \mu \mathrm{s}$, reproducing the input value. In contrast, the obtained "on" time is larger than the input value by a factor of 3 for $50 \mu$ s bin time and by a factor of 6 for $100 \mu \mathrm{s}$ bin time. The analysis based on histograms (Figure 7d) gives similar results: $50 \mu \mathrm{s}$ and $77 \mu \mathrm{s}$ "off" time for this particular trace, respectively. Because of the rapid decay of an exponential, very long observation times are necessary in order to accurately reveal the triplet kinetics if an inadequate binning time is used. ${ }^{9}$ For systems with low $Y_{\text {ISC }}$ and a very short triplet lifetime it thus renders impossible to accurately reveal triplet kinetics under experimental conditions as described here. On the other hand, if a molecule can be followed for a very long period of time, the triplet lifetime can be determined even with a binning time that is longer than the actual "off" times.

Table 2 summarizes the results of the analysis of the MCsimulated transients. The given errors are standard deviations obtained by averaging the results of 50 transients each, with a times per bin of 50 and $100 \mu \mathrm{s}$, respectively. While the "off" times are accurately revealed also in the case of a binning time longer than the actual "off" duration, there is a considerable over-estimation of the "on" time. It can therefore be concluded that the actual "on" times in the experiments are shorter than those estimated from the experimental data, and consequently, the actual $Y_{\text {ISC }}$ values are larger than those obtained from our analysis.

The deviations of the values obtained by the histogram method for the short bin time $(50 \mu \mathrm{s})$ reflect the peculiarity to determine "on" and "off" levels if the shot noise of photon counting becomes comparable to the difference between both intensity levels. It shows the superiority of the ACF over the histogram method as recently pointed out by Verberk and Orrit. $^{30}$

\section{Summary and Conclusion}

The "on"- "off" fluorescence intermittency of isolated PMI chromophores on different time scales was studied by timeresolved confocal fluorescence detection. The short "off" times with an average duration between several tens to hundreds of 
microseconds are attributed to triplet excursions of the single quantum system. This assignment is evidenced by the shortening of the "off" times in the presence of molecular oxygen and by the exponential decay of the "off" time distribution. Only a weak dependence of the triplet kinetics on the polymer matrix is found under an argon atmosphere. The molecule-to-molecule variations of the triplet lifetime indicate heterogeneity of the matrix in which the molecules are embedded.

The distribution of the longer "off" times ranging up to seconds follows a power law. Although a detailed understanding of the mechanism underlying the fluorescence intermittency process requires further investigation, from the present results it can already be concluded that there must be a wide distribution of transition rates from the dark state back to the fluorescent state. Additional experimental effort has to be undertaken to find direct evidence for the assumption of radical ion formation in this system. Temperature-dependent experiments might allow for distinguishing between tunneling and thermally activated processes. We hope nevertheless that the finding of a power law "off"-time distribution will encourage efforts toward a unified picture of single-molecule and semiconductor nanocrystal blinking behavior.

Acknowledgment. This work was supported by the "Sonderforschungsbereich 625" (DFG) and by the "Zentrum für multifunktionelle Werkstoffe und miniaturisierte Funktionseinheiten" (BMBF 03N 6500).

\section{References and Notes}

(1) Moerner, W. E. Science 1997, 277, 1059.

(2) Bernard, J.; Fleury, L.; Talon, H.; Orrit, M. J. Chem. Phys. 1993, 98,850 . 32.

(4) Ha, T.; Enderle, T.; Chemla, D. S.; Selvin, P. R.; Weiss, S. Chem. Phys. Lett. 1997, 271, 1.

(5) Mais, S.; Tittel, J.; Basché, T.; Bräuchle, C.; Gohde, W.; Fuchs, H.; Muller, G.; Müllen, K. J. Phys. Chem. A 1997, 101, 8435.

(6) Shuming, N.; Chiu, D. T.; Zare, R. N. Science 1994, 266, 1018.
(7) Yip, W. T.; Hu, D. H.; Yu, J.; Vanden Bout, D. A.; Barbara, P. F. J. Phys. Chem. A 1998, 102, 7564

(8) Veerman, J. A.; Garcia Parajo, M. F.; Kuipers, L.; van Hulst, N. F. Phys. Rev. Lett. 1999, 83, 2155.

(9) Hübner, C. G.; Renn, A.; Renge, I.; Wild, U. P. J. Chem. Phys. 2001, 115, 9619.

(10) English, D. S.; Furube, A.; Barbara, P. F. Chem. Phys. Lett. 2000, 324,15 .

(11) Tinnefeld, P.; Buschmann, V.; Weston, K. D.; Sauer, M. J. Phys Chem. A 2003, 107, 323.

(12) Weston, K. D.; Buratto, S. K. J. Phys. Chem. A 1998, 102, 3635.

(13) Hou, Y. W.; Higgins, D. A. J. Phys. Chem. B 2002, 106, 10306.

(14) Ambrose, W. P.; Goodwin, P. M.; Martin, J. C.; Keller, R. A. Phys. Rev. Lett. 1994, 72, 160.

(15) Christ, T.; Petzke, F.; Bordat, P.; Herrmann, A.; Reuther, E.; Müllen, K.; Basché, T. J. Lumin. 2002, 98, 23.

(16) Lu, H. P.; Xie, X. S. J. Phys. Chem. B 1997, 101, 2753.

(17) Sauer, M.; Drexhage, K. H.; Lieberwirth, U.; Muller, R.; Nord, S.; Zander, C. Chem. Phys. Lett. 1998, 284, 153.

(18) Vargas, F.; Hollricher, O.; Marti, O.; de Schaetzen, G.; Tarrach, G. J. Chem. Phys. 2002, 117, 866 .

(19) Christ, T.; Kulzer, F.; Bordat, P.; Basché, T. Angew. Chem., Int. Ed. 2001, 40, 4192.

(20) Hofkens, J.; Maus, M.; Gensch, T.; Vosch, T.; Cotlet, M.; Kohn, F.; Herrmann, A.; Müllen, K.; De Schryver, F. J. Am. Chem. Soc. 2000, $122,9278$.

(21) Kohn, F.; Hofkens, J.; Gronheid, R.; Van der Auweraer, M.; De Schryver, F. C. J. Phys. Chem. A 2002, 106, 4808.

(22) Vosch, T.; Cotlet, M.; Hofkens, J.; Van der Biest, K.; Lor, M.; Weston, K.; Tinnefeld, P.; Sauer, M.; Latterini, L.; Müllen, K.; De Schryver, F. C. J. Phys. Chem. A 2003, 107, 6920.

(23) Hübner, C. G.; Zumofen, G.; Renn, A.; Herrmann, A.; Müllen, K.; Basché, T. Phys. Rev. Lett. 2003, 91, 093903.

(24) Weston, K. D.; Carson, P. J.; DeAro, J. A.; Buratto, S. K. Chem. Phys. Lett. 1999, 308, 58.

(25) Fleury, L.; Segura, J. M.; Zumofen, G.; Hecht, B.; Wild, U. P. Phys. Rev. Lett. 2000, 84, 1148.

(26) Kuno, M.; Fromm, D. P.; Hamann, H. F.; Gallagher, A.; Nesbitt, D. J. J. Chem. Phys. 2001, 115, 1028

(27) Shimizu, K. T.; Neuhauser, R. G.; Leatherdale, C. A.; Empedocles, S. A.; Woo, W. K.; Bawendi, M. G. Phys. Rev. B 2001, 6320, art. no. 205316

(28) Zondervan, R.; Kulzer, F.; Orlinskii, S. B.; Orrit, M. J. Phys. Chem. A 2003, 107, 6770.

(29) Holman, M. W.; Liu, R. C.; Adams, D. M. J. Am. Chem. Soc. 2003 , 125,12649 .

(30) Verberk, R.; Orrit, M. J. Chem. Phys. 2003, 119, 2214. 